\title{
REDES EM CIÊNCIA DA INFORMAÇÃO: EVIDÊNCIAS COMPORTAMENTAIS DOS PESQUISADORES E TENDÊNCIAS EVOLUTIVAS DAS REDES DE CO- AUTORIA
}

\author{
Wladmir Cardoso Brandão \\ Fernando Silva Parreiras \\ Antonio Braz de Oliveira e Silva
}

\begin{abstract}
Resumo
A análise de redes complexas, em especial das redes sociais, tem despertado o interesse da comunidade científica mundial. $O$ interesse decorre de sua capacidade de representação de problemas complexos de maneira objetiva, oferecendo um arcabouço teórico e prático para o estudo das características, propriedades e comportamentos dos elementos e relações que compõem os problemas. No campo da ciência da informação, técnicas de análise de redes sociais vêm sendo utilizadas para analisar o fluxo de informação em comunidades e a estrutura das redes de colaboração científica. No entanto, aspectos evolutivos, que poderiam auxiliar na predição do comportamento dos atores e, conseqüentemente, da estrutura futura da rede, tem sido negligenciados. Pode-se comparar a análise de redes sociais, desconsiderando o aspecto evolutivo, à análise de uma fotografia, estática por natureza: apesar de importante, oferece menor riqueza de detalhes que um filme onde elementos temporais facilitam a absorção do contexto e a construção de
\end{abstract}

\section{INTRODUÇÃO}

O conceito de rede é aplicado em diversas áreas do conhecimento humano. Genericamente, pode-se definir uma rede como um conjunto de elementos que mantêm conexões uns com os outros. Na litera- cenários para predição. O presente artigo evidencia o comportamento dos pesquisadores do campo de ciência da informação no Brasil, recorrendo à análise da evolução de redes de co-autoria na área, apresentando tendências que provocarão impactos na estrutura dessas redes nos próximos anos.

\section{Palavras-Chave}

Redes sociais, análise de rede social, redes de co-autoria, ciência da informação.

tura matemática, as redes são reconhecidas como grafos, seus elementos como vértices e suas conexões como arestas. Já nas ciências sociais, os elementos são denominados atores e suas conexões são laços. Já na literatura da ciência da compu- 
tação, os elementos são reconhecidos como nós e as conexões como ligações.

No mundo real, sistemas podem ser representados e problemas podem ser tratados através da abordagem de rede. Um grupo de pessoas em uma organização trocando mensagens eletrônicas a fim de desempenhar suas funções pode ser interpretado como uma rede social, onde cada pessoa passa a ser um ator e as mensagens eletrônicas por eles trocadas passam a ser os laços da rede. Nesse sentido, o entendimento das redes, de sua estrutura, características e comportamento é fundamental para a compreensão das diversas classes de sistemas e problemas que podem ser por elas modelados e tratados.

Em redes simples, com dezenas ou centenas de nós e ligações, a própria visualização e interpretação do grafo a olho nu se constitui em importante ferramenta de análise. Entretanto, a modelagem da grande maioria dos sistemas e problemas reais envolve redes complexas, com milhares ou mesmo milhões de nós e ligações. Além disso, os nós em redes complexas podem assumir diferentes formas e apresentarem diferentes atributos, e as ligações podem ter significados diferentes podendo assumir valores (ponderação) e terem orientação (direcionamento). Para essa classe de rede, a análise a olho nu se torna de pouca valia, uma vez que a quantidade de informação é tão grande que inviabiliza sua completa visualização e torna praticamente impossível seu processamento visual pelo cérebro humano.

\subsection{MODELOS E REDES REAIS}

Uma gama enorme de sistemas e problemas do mundo real podem ser modelados como redes complexas. A internet, a malha rodoviária e ferroviária e o sistema de distribuição de energia de um país podem ser interpretados como redes tecnológicas complexas. A web, assim como as redes de citação entre artigos acadêmicos e os thesaurus ${ }^{1}$ podem ser entendidos como complexas redes de informação (ou conhecimento) uma vez que sua estrutura reflete a estrutura de armazenamento de informação em seus elementos. Sistemas biológicos também podem ser representados por redes. Um exemplo é a cadeia predatória entre animais onde os nós representam as diversas classes de animais presentes na fauna e as ligações representam uma relação de predação entre duas classes.

Ao longo dos anos modelos matemáticos foram desenvolvidos visando prover métodos e mecanismos para análise de redes complexas. Modelos de geração e crescimento de redes têm sido propostos e suas propriedades têm sido estudadas. Dentre as propriedades, cabe-se destacar

\footnotetext{
${ }^{1}$ Vocabulários controlados utilizados para indexação de documentos em bibliotecas.
} 
aquelas relacionadas ao tamanho da rede (diâmetro ou diameter), graus de centralidade (grau ou degree centrality, grau de intermediação ou beetweenness centrality, grau de proximidade ou closeness centrality), grau de transitividade (coeficiente de agrupamento ou clustering coefficient) e às suas respectivas distribuições.

Modelos de geração criam redes que apresentam características particulares no que tange a algumas de suas propriedades. Paul Erdös e Alfréd Rényi (ERDÖs; RÉNYI 1959, 1960) propuseram a geração de uma rede a partir de ligações estabelecidas de maneira aleatória entre seus nós, ou seja, todos os nós da rede têm a mesma probabilidade de estabelecerem relações uns com os outros. Tal modelo acaba por gerar uma rede denominada rede randômica ou aleatória, com diâmetro pequeno e baixo coeficiente de agrupamento.

Duncan Watts e Steve Strogatz (WATTS; STROGATZ, 1998), baseando-se no famoso experimento de Stanley Milgram da década de 1960, denominado posteriormente por "Six Degrees of Separation" (GUARE, 1990), propõem um modelo de geração de rede a partir da reescrita ou adição de um pequeno número de ligações de maneira aleatória em uma rede regular ${ }^{2}$, o que acaba por gerar uma rede do tipo mundo pequeno ("small-world") com pe-

\footnotetext{
${ }^{2}$ Redes onde cada nó está conectado a um número $k$ de vizinhos.
}

queno diâmetro, porém com elevado coeficiente de agrupamento.

No modelo de crescimento proposto por Albert-László Barabási e Réka Albert (BARABÁSI; ALBERT, 1999), nós entrantes na rede se associam as nós presentes por regras de preferência ("preferential attachment'), sendo que os nós com maior número de ligações têm maior probabilidade de receber novas ligações que os outros. Neste modelo, a distribuição dos graus da rede tende a seguir uma lei de potência onde um número muito pequeno de nós possui muitas ligações (alto "degree centrality") e um grande número de nós possui pouquíssimas ligações (baixo "degree centrality"). Tal modelo é considerado um modelo "power-law" justamente pelo fato de produzir uma distribuição de graus que segue uma lei de potência. As redes que seguem essa distribuição são conhecidas como redes livres de escala ("scalefree networks").

Alguns modelos matemáticos se mostraram adequados para representação de redes reais. Estudos demonstram que a internet (FALOUTSOS; FALOUTSOS; FALOUTSOS, 1999), a web (ADAMIC, 1999), as redes de colaboração científica (NEWMAN, 2001) e as redes de correspondência eletrônica (EBEL; MIELSCH; BORNHOLDT, 2002) apresentam o fenômeno "small-world" (baixo diâmetro e alto coeficiente de agrupamento) e são, de fato, 
redes livres de escala (distribuição do grau dos nós segue uma lei de potência).

\subsection{REDES SOCIAIS}

Para Scott (2000) e Wasserman e Faust (1994), uma rede social é um conjunto de pessoas ou grupos de pessoas com algum padrão de conexão e interação amizade, relações de negócio, relações conjugais - entre elas. Newman (2001) afirma que as ciências sociais são o campo científico com a maior bagagem histórica em estudos quantitativos sobre redes complexas reais. Segundo o autor, ainda nas décadas de 1920 e 1930, Jacob Moreno já estudava padrões de amizade em pequenos grupos de pessoas (MORENO, 1934) e Davis e seus colaboradores estudavam os círculos sociais entre mulheres em pequenas cidades no sul dos Estados Unidos (DAVIS; GARDNER; GARDNER, 1941). Os próprios experimentos de Stanley Milgram podem ser considerados experimentos no campo das ciências sociais.

De acordo com Marsden (1990), os métodos tradicionais de abordagem dos problemas inerentes às redes sociais padeciam de problemas de imprecisão e subjetividade, muito em decorrência do mecanismo de coleta de dados utilizado na época, geralmente questionários ou entrevistas. Atualmente, em virtude da utilização intensiva de mecanismos computacionais, existem diversas fontes de informação que possibilitam coleta automatizada de um volume de dados expressivamente maior que no passado. Além disso, diversos pesquisadores tem se dedicado a desenvolver pesquisas no sentido de identificar e avaliar propriedades estatísticas de redes sociais cada vez maiores em tamanho (AMARAL et al. 2001; NEWMAN; STROGATZ; WATTS 2001; WATTS; STROGATZ, 1998).

No Brasil, parte das pesquisas em redes sociais vem sendo desenvolvidas por pesquisadores do campo da ciência da informação (MATHEUS; SILVA, 2006), seja na análise de fluxos de transferência de informação (MARTELETO, 2001), do compartilhamento de informação e conhecimento em organizações (ALCARÁ et al. 2006; TOMAÉL; ALCARÁ; DI CHIARA 2005; TOMAÉL; MARTELETO 2006) ou no estudo de redes de colaboração científica (PARREIRAS et al. 2006b; SILVA et al. 2006) e de interdisciplinaridade (SILVA et al. 2006a). No entanto, tal como observado por Gueorgi Kossinets e Duncan Watts (KOSSINETS; WATTS, 2006), os estudos atuais tem se limitado a analisar as redes sociais de maneira estática, sem considerar aspectos relacionados à sua evolução ao longo do tempo.

\subsection{MÉTRICAS DE REDE}

Diversas são as métricas adotadas por pesquisadores para a abordagem de 
seus problemas. Existem métricas relacionadas ao tamanho, aos níveis de conectividade e transitividade, ao grau de miscigenação e à estrutura comunitária das redes (NEWMAN, 2003). Não obstante, novas métricas surgem à medida que surgem também novos problemas a serem equacionados.

No entanto, existe um conjunto de métricas formalmente estabelecidas e comumente utilizadas no estudo e resolução de grande parte dos problemas envolvendo redes complexas, sobre as quais cabe uma breve explicação.

Considere um grafo não direcionado $G(N, L)$, onde $N$ representa o conjunto de nós do grafo, sendo definido por $N=\left\{n_{1}\right.$, $\left.n_{2}, \ldots, n_{g}\right\}$ e $L$ representa o conjunto de ligações entre pares de nós no grafo, sendo definido por $L=\left\{I_{1}, I_{2}, \ldots, I_{h}\right\}$. Considere ainda que $g$ represente o número de nós do grafo e $h$ o número de ligações entre pares de nós. Cada ligação em $L$ pode ser representada por:

$$
l_{k}=\left(n_{i}, n_{j}\right) \Rightarrow\left\{\begin{array}{l}
1 \leq k \leq h \\
1 \leq i \leq g \\
1 \leq j \leq g
\end{array}\right\}
$$

Sendo assim, $I_{k}$ corresponde a uma ligação dedicada a conectar o nó $n_{i}$ ao $n_{j}$.

Considere também que o número de nós adjacentes a um nó específico é representado por $d\left(n_{i}\right)$. Dessa forma, torna-se possível definir as seguintes métricas de rede:

Diâmetro da Rede (D) - Medida de tamanho que indica a distância geodésica (geodesic distance) - ou caminho mínimo (shortest path) - média existente entre os pares de nós conectados na rede. Em redes com apenas um componente, considerando $p_{i j}$ como a distância geodésica entre $i$ e j, o valor do diâmetro pode ser expresso da seguinte forma:

$$
D=\frac{1}{g(g+1) / 2} \sum_{i \geq j} p_{i j}
$$

Em redes com mais de um componente, a definição de tal métrica pode se tornar um problema. Newman (2003) apresenta uma solução viável para sua resolução.

\section{Medidas de Centralidade da Rede - Me-} didas indicativas do nível de conectividade direta entre nós da rede. Dentre elas destacam-se:

Grau de Centralidade (DCE) - Número de conexões (de entrada e saída) de cada nó. Equivale ao número de nós adjacentes a um nó e seu valor é dado por:

$$
\operatorname{DCE}\left(n_{i}\right)=d\left(n_{i}\right)
$$

Considerando $n_{\max }$ como o nó de maior grau de centralidade na rede, a medida normalizada é dada por: 


$$
\operatorname{NDCE}\left(n_{i}\right)=\frac{\operatorname{DCE}\left(n_{i}\right)}{\operatorname{DCE}\left(n_{\max }\right)}
$$

Grau de Intermediação (BCE) - Número de distâncias geodésicas entre quaisquer dois nós da rede que passam por um nó específico. Tal medida indica o quanto um nó está no caminho mínimo entre outros dois pares de nós. Seja $p_{j k}$ o número de distâncias geodésicas que ligam os nós $j$ e $k$, e $p_{j k}\left(n_{i}\right)$ o número de distâncias geodésicas que passam pelo nó $n_{i}$. O grau de intermediação é dado por:

$$
B C E\left(n_{i}\right)=\sum_{j<k} \frac{p_{j k}\left(n_{i}\right)}{p_{j k}}
$$

Considerando $n_{\max }$ como o nó de maior grau de intermediação na rede, a medida normalizada é dada por:

$$
\operatorname{NBCE}\left(n_{i}\right)=\frac{B C E\left(n_{i}\right)}{B C E\left(n_{\max }\right)}
$$

Grau de Proximidade (CCE) - Inverso da soma das distâncias geodésicas entre cada nó da rede e os demais. Indica o quão próximo um nó da rede está dos demais. Considerando mais uma vez $\mathrm{p}_{i j}$ como a distância geodésica entre $i$ e $j$, o valor do grau de proximidade pode ser expresso da seguinte forma:

$$
\operatorname{CCE}\left(n_{i}\right)=\left[\sum_{j=1}^{g} p_{i j}\right]^{-1}
$$

Considerando $n_{\max }$ como o nó de maior grau de proximidade na rede, a medida normalizada é dada por:

$$
\operatorname{NCCE}\left(n_{i}\right)=\frac{\operatorname{CCE}\left(n_{i}\right)}{\operatorname{CCE}\left(n_{\max }\right)}
$$

Medidas de Transitividade da Rede Medidas que indicam o nível de conectividade indireta (entre vizinho) da rede.

\section{Coeficiente de Agrupamento (CC1) -}

Indica a probabilidade dos vizinhos de um nó da rede se conectarem entre si. Considerando $N_{i}$ o conjunto de nós vizinhos ao nó $i, k_{i}$ o número de vizinhos de $i$ e $c_{j k}=1$ ou 0 caso exista ou não uma ligação entre $j$ e $k$ desde que $j, k \in N_{i}$, o valor do coeficiente de agrupamento pode ser expresso por:

$$
\operatorname{CC1}\left(n_{i}\right)=\frac{\sum_{j>k} c_{j k}}{k_{i}\left(k_{i}-1\right) / 2}
$$

Considerando $n_{\max }$ como o nó de maior coeficiente de agrupamento na rede, a medida normalizada é dada por:

$$
\operatorname{NCC1}\left(n_{i}\right)=\frac{C C 1\left(n_{i}\right)}{C C 1\left(n_{\max }\right)}
$$

\section{EVOLUÇÃo dA REDE DE CO-} AUTORIA EM CIÊNCIA DA INFORMAÇÃO NO BRASIL

Nas redes de co-autoria, os autores de trabalhos publicados em veículos de difusão de conhecimento científico como 
revistas, jornais e eventos são considerados atores, sendo que, para cada autoria em conjunto entre dois atores é criado um laço relacional entre eles. Diversas redes de co-autoria vem sendo estudadas ao longo dos anos (NEWMAN, 2001). No Brasil

RedeCl

$<$ http://www.redeci.netic.com.br $>$ oferece um importante arcabouço bibliográfico para realização de estudos de co-autoria dentro do campo de ciência da informação (PARREIRAS et al., 2006), uma vez que mantém registros de diversos veículos de difusão, possibilitando a realização de estudos bibliométricos por parte de pesquisadores interessados.

Parreiras e seus colaboradores (PARREIRAS et al., 2006), ao avaliarem as métricas da rede de co-autoria do campo de ciência da informação no Brasil, apontaram a existência de uma baixa colaboração global entre os autores. Por colaboração global entende-se que as sub-redes de autores colaboram pouco entre si, sendo que o maior grau de colaboração é local dentro da própria sub-rede). A presença de um alto número de sub-redes e de autores individuais (que não colaboram com outros autores) fazem com que a colaboração global, medida pela densidade da rede, seja baixa. No entanto, não se pode afirmar que este comportamento esteja variando ao longo do tempo e que a tendência é o aumento ou a diminuição da colaboração global, apesar de haver indícios de que a topologia da rede não se altera de maneira significativa ao longo do tempo.

Para identificar o padrão comportamental dos pesquisadores da área de ciência da informação no Brasil e traçar tendências do perfil de colaboração do campo, foram coletados dados bibliográficos de diversas publicações, conforme mostrado na Tabela 1 abaixo:

Tabela 1 - Coleção de publicações consideradas para análise

\begin{tabular}{|c|c|c|c|c|c|}
\hline \multirow{2}{*}{ Publicações } & \multicolumn{2}{|c|}{ Ano } & \multirow{2}{*}{\begin{tabular}{|c|} 
Volumes e \\
Números
\end{tabular}} & \multirow{2}{*}{ Edições } & \multirow{2}{*}{ Artigos } \\
\hline & Primeiro & último & & & \\
\hline Ciência da Informação & 1996 & 2006 & $25 / 1$ a $35 / 3$ & 32 & 366 \\
\hline CINFORM & 2003 & 2005 & 4 a 6 & 3 & 127 \\
\hline DatagramaZero & 1999 & 2006 & $1 / 0$ a $7 / 3$ & 40 & 183 \\
\hline ENANCIB & 1996 & 2006 & 3 a 7 & 5 & 469 \\
\hline Encontros Bibli & 1996 & 2006 & 1 a 21 & 21 & 84 \\
\hline Informação e Sociedade & 1996 & 2005 & $6 / 1$ a $16 / 2$ & 19 & 187 \\
\hline Informática Pública & 1996 & 2006 & $1 / 1$ a $8 / 1$ & 14 & 67 \\
\hline Perspectivas em Ciência da Informação & 1996 & 2006 & $1 / 1$ a $11 / 1$ & 23 & 172 \\
\hline Revista Digital de Biblioteconomia e Ciência da Informação & 2003 & 2006 & $1 / 1$ a $3 / 2$ & 6 & 39 \\
\hline Transinformação & 2002 & 2006 & $14 / 2$ a $18 / 3$ & 14 & 118 \\
\hline TOTAL & - & - & - & 177 & 1812 \\
\hline
\end{tabular}

Conforme se observa, foram consideradas, para efeito de análise, 10 publicações (8 periódicos e 2 publicações vincula das à eventos) com a característica comum de disponibilizarem na internet a íntegra de seus 1812 artigos. Os dados da coleção 
considerada representam 177 edições de publicações durante um período de 11 anos, de 1996 à 2006.

Para considerar o aspecto evolutivo, a coleção foi dividida em 5 faixas temporais todas elas compostas por artigos das 10 publicações supra apresentadas. A primeira faixa compreende o período de três anos entre 1996 a 1998, sendo que as faixas subseqüentes agregam, a cada faixa anterior, períodos de 2 anos. O número de artigos e de autores na coleção por faixa temporal é apresentado no Quadro 2 abaixo:

\begin{tabular}{|c|c|c|}
\hline Faixa Temporal & $\mathbf{N}^{\circ}$ de Artigos & $\mathbf{N}^{\circ}$ de Autores \\
\hline 1996 a 1998 & 301 & 368 \\
\hline 1996 a 2000 & 503 & 566 \\
\hline 1996 a 2002 & 755 & 804 \\
\hline 1996 a 2004 & 1284 & 1281 \\
\hline 1996 a 2006 & 1812 & 1725 \\
\hline
\end{tabular}

Quadro 2 - Número de artigos e autores por faixa temporal

A subdivisão da coleção em faixas temporais cumulativas permite a captação de tendências topológicas e comportamentais da rede de colaboração de manei- ra mais efetiva do que a análise estática considerando apenas uma dimensão temporal fixa.

Em uma primeira análise e, de acordo com o gráfico A da Figura 1, é possível observar que o número de autores individuais (que publicam sozinhos) é bem superior ao número de autores com co-autoria, o que evidencia o baixo nível de colaboração entre os autores do campo. Entretanto, observa-se também que a diferença entre autores individuais e autores com coautoria vem caindo ao longo do tempo o que evidencia uma tendência de que o comportamento individualista dará lugar a um comportamento mais colaborativo (os resultados que deram origem a esses gráficos encontram-se no Apêndice I).

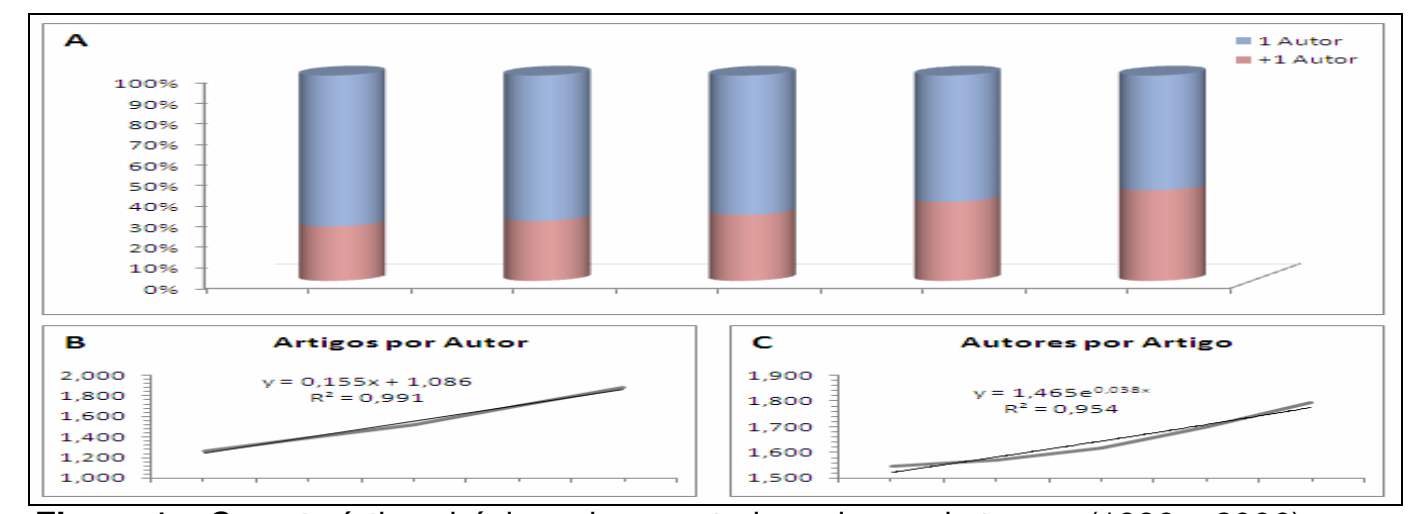

Figura 1 - Características básicas de co-autoria ao longo do tempo (1996 a 2006) 
Essa mudança comportamental pode ser explicada pela presença de um número cada vez maior de cursos de pósgraduação e pela característica colaborativa da relação orientando-orientador - de produzirem em conjunto - ter se intensificado (SILVA et al., 2006).

Os gráficos B e C da Figura 1 apresentam, respectivamente, a média de artigos por autor, refletindo a produtividade, e a média de autores por artigo, refletindo a colaboração, ao longo do tempo. Nota-se que a produtividade dos autores vêm crescendo de maneira linear enquanto o nível de colaboração também vêm crescendo, porém numa progressão exponencial. Apesar do ritmo de crescimento da colaboração ser exponencial, o coeficiente da potência apresenta baixo valor de 0,038 demonstrando que o crescimento ainda será baixo nos próximos anos - para se dobrar o número médio de autores, atualmente uma pouco acima de 1,7 por artigo, seria necessário, com a tendência atual, cerca de 34 anos (lembrando que as bases são bianuais).

Ao se analisar as redes sociais decorrentes da co-autoria entre autores em cada uma das faixas temporais, é possível observar que a tendência é de queda na densidade da rede como um todo, conforme apresentado no gráfico $\mathrm{A}$ da Figura 2, e de estabilidade no coeficiente de agrupamento (gráfico B, Figura 2) e no diâmetro da rede (gráfico C, Figura 2).

Apesar de parecer contraditório o fato do gráfico $C$ da Figura 1 ter demonstrado que a colaboração está aumentando e do gráfico $\mathrm{A}$ da Figura 2 ter demonstrado 0 contrário, trata-se, na verdade, de duas situações distintas. Como era de se esperar, enquanto o nível de colaboração local vem subindo, em decorrência do aumento na colaboração dentro das sub-redes por parte dos autores "veteranos", o nível de colaboração global (na rede como um todo) vem caindo uma vez que autores "novatos" entram na rede a cada ano apresentando, no momento de sua entrada, baixo grau de colaboração.

A forte elevação do diâmetro da rede na faixa temporal de 1996 a 2004 pode ser explicada pelo comportamento denominado "brokerage", isto é, a capacidade de se saltar buracos na estrutura social ("structural holes") entre grupos distintos (BURT, 1995), onde pontes não existentes entre duas ou mais sub-redes são criadas a partir da colaboração entre grupos de autores que antes não colaboravam entre si.

Nota-se que, já no momento seguinte, com a entrada de novos autores e a presença de laços relacionais entre eles, a rede voltou a apresentar padrões equivalentes aos anteriores. 
Já a forte queda no coeficiente de agrupamento na faixa temporal de 1996 a 2006 pode ser explicada pelo elevado número de novos autores que entraram publicando artigos entre 2005 e 2006 de maneira dispersa, não contribuindo para a constituição de sub-redes densas de co-autoria.

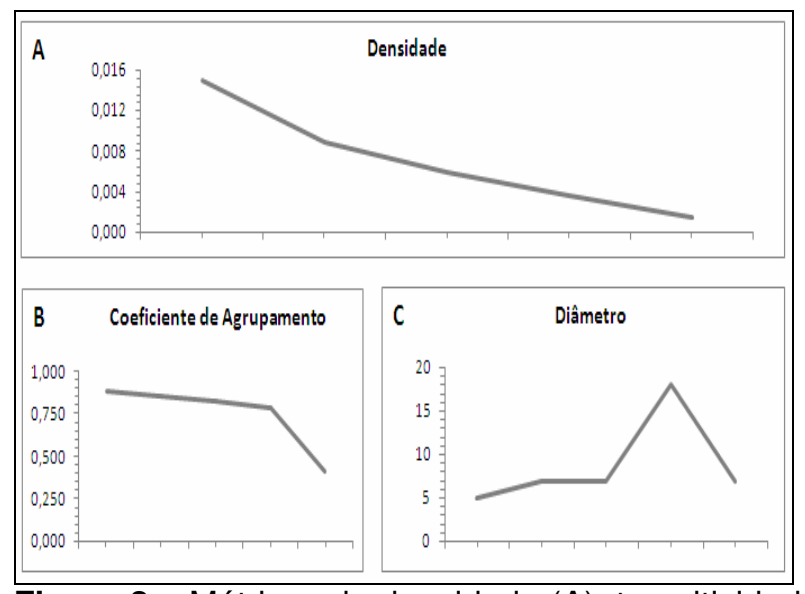

Figura 2 - Métricas de densidade (A), transitividade (B) e tamanho (C) da rede ao longo do tempo (1996 a 2006)
Outro aspecto importante analisado foi a evolução da distribuição dos graus de centralidade dos atores da rede de coautoria ao longo do tempo. Tal análise permitiu a caracterização da rede de coautoria em ciência da informação como uma rede livre de escala ("scale-free network'), o que, por si só, já oferece subsídios para tirar conclusões sobre suas propriedades, topologia e padrão comportamental, como a presença de regras de preferência ("preferential attachment"). Conforme pode ser observado na Figura 3 , a distribuição dos graus de centralidade da rede em cada faixa temporal segue uma lei de potência ("power-law").

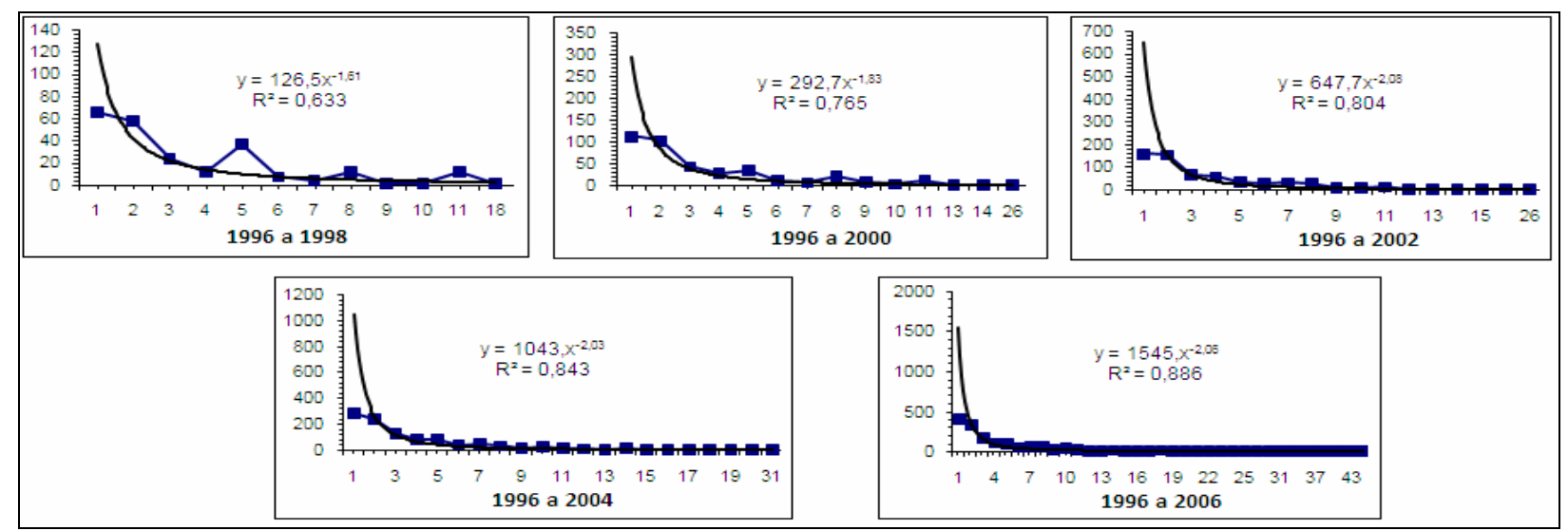

Figura 3 - Distribuição de graus de centralidade da rede por faixa temporal

Cabe também salientar que, a distribuição dos graus observada não se altera significativamente ao longo do tempo, fato que pode ser comprovado através da análise dos coeficientes de correlação de $\mathrm{Pe}$ arson calculado entre os resultados estimados e o valor observado para o último período considerado período (1996-2006).
Nesse caso, os coeficientes apresentados na Tabela 2, correlacionam os valores observados no último período para a distribuição de graus com as estimativas feitas a partir das equações das leis de potência para os cinco períodos considerados. Observa-se que os coeficientes são bastante próximos, o que demonstra a similaridade da distribuição dos graus em 
da distribuição dos graus em todas os períodos e, conseqüentemente, a sua baixíssima mutabilidade ao longo do tempo. As alterações na distribuição de graus, ao lon- go do tempo, com a entrada de novos autores não altera as características da rede.

Tabela 2 - Coeficiente de correlação de Pearson para a distribuição de graus de centralidade da rede de co-autoria

\begin{tabular}{|c|c|c|c|c|c|c|}
\hline & $\begin{array}{c}1996 \text { a } 2006 \\
\text { Real }\end{array}$ & $\begin{array}{c}1996 \text { a } 2006 \\
\text { Regressão }\end{array}$ & $\begin{array}{c}1996 \text { a } 2004 \\
\text { Regressão }\end{array}$ & $\begin{array}{c}1996 \text { a } 2002 \\
\text { Regressão }\end{array}$ & $\begin{array}{c}1996 \text { a } 2000 \\
\text { Regressão }\end{array}$ & $\begin{array}{c}1996 \text { a } 1998 \\
\text { Regressão }\end{array}$ \\
\hline 1996 a 2006 - Real & 1,000 & & & & & \\
\hline 1996 a 2006 - Regressão & 0,862 & 1,000 & & & & \\
\hline 1996 a 2004 - Regressão & 0,865 & 1,000 & 1,000 & & & \\
\hline 1996 a 2002 - Regressão & 0,859 & 1,000 & 1,000 & 1,000 & & \\
\hline 1996 a 2000 - Regressão & 0,886 & 0,999 & 0,999 & 0,998 & 1,000 & \\
\hline 1996 a 1998 - Regressão & 0,910 & 0,994 & 0,995 & 0,993 & 0,998 & 1,000 \\
\hline
\end{tabular}

* Todos os coeficientes de correlação são significativos ao nível 0,01

\section{CONCLUSÕES}

A utilização de aspectos evolutivos da rede de co-autoria em ciência da informação no Brasil viabilizou a realização de análises aprofundadas sobre as propriedades e características topológicas da rede e sobre os padrões comportamentais dos atores envolvidos.

Ficou evidenciado que a rede em questão pode ser considerada uma rede do tipo mundo-pequeno ("small-world"), uma vez que apresenta diâmetro pequeno - de amanho 7 - e alto nível de transitividade medido pelo coeficiente de agrupamento ("clustering coefficient"). Além disso, a distribuição dos graus de centralidade segue uma lei de potência evidenciando a existência do comportamento de associação preferencial ("preferential attachment") de seus atores, ou seja, autores muito colaborativos tendem a colaborar mais do que aqueles pouco colaborativos. Além disso, comprovou-se a tendência futura de queda da densidade da rede, o que permite afirmar que a colaboração global tende a diminuir com o passar dos anos. Entretanto, há evidências de que a colaboração local, dentro das sub-redes, tende a aumentar, embora em ritmo lento.

As acentuadas variações de diâmetro e de coeficiente de agrupamento ocorridas em algumas faixas temporais sugerem que em redes de baixa densidade e com vários atores isolados, pequenas alterações no comportamento individual com relação à colaboração podem alterar significativamente a topologia da rede. Passa a ser um problema de pesquisa identificar as características e atributos do autor que fez a ligação entre dois grupos antes isolados, conforme o ponto seis destacado entre as preocupações futuras. 
Como é comum na ciência, a resposta a uma questão produz diversas outras questões a serem investigadas. Sendo assim, cabe ressaltar a importância de, em trabalhos futuros: 1) Verificar a influência de aspectos geográficos nas práticas de colaboração entre as sub-redes; 2) Avaliar a existência de correlações entre a produtividade e a colaboração dos autores utilizando métricas associadas aos aspectos topológicos da rede; 3) Estudar o comportamento da rede em função de conceito de autores produtivos combinando métricas de produção, colaboração e tempo de permanência na rede; 4) Verificar a influência dos atributos dos atores como status, filiação e tempo de permanência sobre a transitividade da rede; 5) Verificar a possibilidade de identificação de diferenças comportamentais entre pesquisadores de diversas áreas de concentração a partir das características colaborativas observadas na rede; 6) Identificar se o estabelecimento de colaboração entre grupos na rede segue um padrão acidental ou proposital por parte dos brokers (atores que mantêm sub-redes conectadas) da rede.

\section{REFERÊNCIAS}

ADAMIC $_{1}$ Lada. The small world web. In.: EUROPEAN CONFERENCE IN DIGITAL LIBRARIES, 3., 1999. Proceedings... Paris: ECDL, 1999. p.443-454.

ALCARÁ, Adriana Rosecler et al. As redes sociais como instrumento estratégico para a inteligência competitiva. Transinformação, Campinas, v.18, n.2, p.143-153, 2006.

AMARAL, L.A.N. et al. Classes of smallworld networks. Proceedings of National Academy of Science, Washington, v.97, n.21 p.1149-11152, 10 Oct. 2000.

BARABÁSI, Albert-László; ALBERT, Réka. Emergence of scaling in random networks. Science. Washington, n.286, p.509-512, 1999.

BURT, Ronald S. Structural holes: the social structure of competition. Cambridge: Harvard University Press, 1995.

DAVIS, A.; GARDNER, B.B.; GARDNER, M.R. Deep South. Chicago: University of Chicago Press, 1941.

EBEL, Holger; MIELSCH, Lutz-Ingo; BORNHOLDT, Stefan. Scale-free topology of e-mail networks. Physical Review E, Ridge, v.66, 2002.

ERDÖS, Paul; RÉNYI, Alfréd. On random graphs. Publicationes Mathematicae, Hungria, n.6, p.290-297, 1959.

ERDÖS, Paul; RÉNYI, Alfréd. On the evolution of random graphs. Publications of the Mathematical Institute of the Hungarian Academy of Sciences, Hungria, n.5, p.1761, 1960.

FALOUTSOS, Michalis; FALOUTSOS, Petros; FALOUTSOS, Christos. On power-law relationships of the Internet topology. Computer Communication Review, New York, v.29, p.251-262, 1999.

GUARE, J. Six Degrees of separation: a play. New York: Vintage Books, 1990.

KOSSINETS, Gueorgi; WATTS, Duncan. Empirical Analysis of an Envolving Social Network. Science, Washington, v.311, p.88-90, 2006.

MARSDEN, P.V. Network data and measurement. Annual Review of Sociology, Palo Alto, n.16, p.453-463, 1990. 
MARTELETO, Regina Maria. Análise de redes sociais - aplicações nos estudos de transferência da informação. Ciência da Informação, Brasília, v.30, n.1, p.71-81, 2001.

MATHEUS, Renato Fabiano; SILVA, Antonio Braz de Oliveira e. Análise de redes sociais como método para a Ciência da Informação. DataGramaZero, Rio de Janeiro, v.7, n.2, abr.2006. Disponível em: $<$ http://www.dgz.org.br/abr06/Art 03.htm>.

MORENO, Jacob L. Who Shall Survive? New York: Beacon House, 1934.

NEWMAN, M.E.J. The structure of scientific collaboration networks. Proceedings of The National Academy of Sciences, Washington, v.98, n.2, p.404-409, 2001.

NEWMAN, M. E. J. The structure and function of complex networks. Society for Industrial and Applied Mathematics - SIAM Review. Philadelphia, v.45, n.2, p.167-256, 2003.

NEWMAN, M.E.J.; STROGATZ, Steve; WATTS, Duncan. Random graph with arbitrary degree distributions and their applications. Physics Review E, Ridge, v.64, p.26118, 2001.

PARREIRAS, Fernando Silva et al. REDECI: colaboração e produção científica em ciência da informação no Brasil. Perspectivas em Ciência da Informação, Belo Horizonte, v.11, n.3, p.302-317, 2006.

SCOTT, J. Social Network Analysis: a handbook. 2.ed. London: Sage Publications, 2000.
SILVA, Antonio Braz de Oliveira e et al. Análise de redes sociais como metodologia de apoio para discussão da interdisciplinaridade na ciência da informaç̧ão. Ciência da Informação, Brasília, v.35, n.1, p.72-93, 2006. (a).

SILVA, Antonio Braz de Oliveira e et al. Estudo da rede de co-autoria e da interdisciplinaridade na produção científica com base nos métodos de análise de redes sociais: avaliação do caso do programa de pós-graduação em ciência da informação PPGCI/UFMG. Encontros Bibli, Florianópolis, n.esp., $1^{\circ}$ sem. 2006. (b).

TOMAÉL, Maria Inês; ALCARÁ, Adriana Rosecler; DI CHIARA, Ivone Guerreiro. Das redes sociais à inovação. Ciência da informação, Brasília, v.34, n.2, p.93-104, 2005.

TOMAÉL, Maria Inês; MARTELETO, Regina Maria. Redes Sociais: posições dos atores no fluxo da informação. Encontros Bibli. Florianópolis, .esp., 1ํㅗ. 2006.

WASSERMAN, S.; FAUST, K. Social Network Analysis. Cambridge: Cambridge University Press, 1994.

WATTS, Duncan; STROGATZ, Steve. Collective dynamics of 'small-world' networks. Nature, London, n.393, p.440-442, 1998. 


\section{Apêndice I - Números anuais da coleção considerada para análise}

\begin{tabular}{|c|c|c|c|c|c|c|c|c|c|c|}
\hline \multirow{3}{*}{ Ano } & \multicolumn{6}{|c|}{ Artigos } & \multicolumn{2}{|c|}{ Autores } & \multirow{3}{*}{$\begin{array}{c}\text { Autores por } \\
\text { Artigo }\end{array}$} & \multirow{3}{*}{$\begin{array}{l}\text { Artigos por } \\
\text { Autor }\end{array}$} \\
\hline & \multicolumn{3}{|c|}{ No Ano } & \multicolumn{3}{|c|}{ Acumulado } & \multirow{2}{*}{ No Ano } & \multirow{2}{*}{ Acumulado } & & \\
\hline & 1 Autor & +1 Autor & Total & 1 Autor & +1 Autor & Total & & & & \\
\hline 1996 & 45 & 16 & 61 & 45 & 16 & 61 & 85 & 85 & 1,541 & 1,106 \\
\hline 1997 & 136 & 42 & 178 & 181 & 58 & 239 & 229 & 290 & 1,481 & 1,221 \\
\hline 1998 & 40 & 22 & 62 & 221 & 80 & 301 & 105 & 368 & 1,545 & 1,264 \\
\hline 1999 & 72 & 30 & 102 & 293 & 110 & 403 & 143 & 469 & 1,536 & 1,320 \\
\hline 2000 & 63 & 37 & 100 & 356 & 147 & 503 & 145 & 566 & 1,569 & 1,394 \\
\hline 2001 & 74 & 49 & 123 & 430 & 196 & 626 & 191 & 695 & 1,612 & 1,452 \\
\hline 2002 & 82 & 47 & 129 & 512 & 243 & 755 & 191 & 804 & 1,617 & 1,519 \\
\hline 2003 & 182 & 146 & 328 & 694 & 389 & 1083 & 436 & 1095 & 1,662 & 1,644 \\
\hline 2004 & 96 & 105 & 201 & 790 & 494 & 1284 & 326 & 1281 & 1,701 & 1,705 \\
\hline 2005 & 127 & 164 & 291 & 917 & 658 & 1575 & 415 & 1511 & 1,751 & 1,825 \\
\hline 2006 & 96 & 141 & 237 & 1013 & 799 & 1812 & 371 & 1725 & 1,795 & 1,886 \\
\hline TOTAL & 1013 & 799 & 1812 & 1013 & 799 & 1812 & - & 1725 & 1,795 & 1,886 \\
\hline
\end{tabular}

Wladmir Cardoso Brandão Mestre em ciência da informação pela UFMG, gerente de TI da TRÓPICA Tecnologia e Sistemas, especialista em gestão empresarial pela UFMG, bacharel em ciência da computação pela PUC-MINAS, sócio fundador do NETIC $<$ http://www.netic.com.br>. wbrandao@netic.com.br

Fernando Silva Parreiras Doutorando em ciência da computação pela Universidade Koblenz-Landau (Alemanha), bolsista CAPES, mestre em ciência da informação pela UFMG, bacharel em ciência da computação pela FUMEC, sócio fundador do NETIC. fparreiras@netic.com.br

Antonio Braz de Oliveira e Silva Doutor em ciência da informação pela UFMG, analista do IBGE-MG, sócio fundador do

NETIC, abraz@netic.com.br; antoniobraz.silva@ibge.gov.br

\section{Title}

Networks in Information Science: behavioral evidences of the researchers and evolutional trends of the coauthor network.

\section{Abstract}

The analysis of complex networks, in special of the social networks, has being of interest to the scientific community worldwide. Such interest is due to its ability to represent complex problems in an objective manner, allowing for a theoretical and practical framework to study the characteristics, properties and behavior of the elements composing the problems. In the field of Information Science, techniques of Social Network Analysis have being used to analyze the information flow inside communities as well as the structure of scientific collaboration networks. However, evolutional aspects that could be help to predict the behavior of the authors and, consequently, of the future structure have been neglected. It is possible to compare Social Network Analysis, disregarding its evolutional aspect, with a photograph, which is naturally static. Despite its importance, the photograph cannot be so rich in details as a motion picture, whose temporal elements ease the context analysis and the construction of predicting scenarios. This paper evidences the behavior of researchers in the Information Science's field in Brazil, appealing to the evolutional analysis of the coauthor network, forecasting impacts on the structure of such networks in the next few years.

\section{Keywords}

Social network, social network analysis, coauthor network, information science. 


\section{Título}

Redes en la Ciencia de la Información: evidencias comportamentales de los investigadores y tendencias evolutivas de las redes de coautoría.

\section{Resumen}

La análisis de redes complejas, en especial de las redes sociales, tiene despertado el interés de la comunidad científica mundial. Tal interés viene de su capacidad de representación de problemas complejos de manera objetiva, que ofrece un sistema teórico y práctico para el estudio de las características, propriedades y comportamientos de los elementos y relaciones que componen los problemas. En el campo de la ciencia de la información, técnicas de análisis de redes sociales vienen siendo utilizadas para analizar el flujo de información en comunidades y la estructura de las redes de colaboración científica. Sin embargo, aspectos evolutivos que podrían auxiliar en la predicción del comportamiento de los atores y, consecuentemente, de la estructura futura de la red tiene sido tratados descuidadamente. Se puede comparar a análisis de redes sociales, desconsiderando su aspecto evolutivo, con la análisis de una fotografía, estática por naturaleza. A pesar de importante, ela ofrece menor riqueza de detalles que una película donde elementos temporales facilitan la absorción del contexto y la construcción de ambientes para predicción. El presente artículo evidencia el comportamiento de los investigadores del campo de la ciencia de la información brasileña, recorriendo a la análisis de la evolución de redes de coautoría en el campo, pronosticando impactos en la estructura de estas redes en los próximos años.

\section{Palabras Clave}

Redes sociales, análisis de red social, red de coautoría, ciencia de la información. 